\title{
Retrospective survey of avian influenza H5N1 infection in Northern Vietnam by using a combinational serologic assay
}

\author{
Hoa Minh Luong ${ }^{1,2^{\star}}$, Sheng-Fan Wang ${ }^{3,4,5^{*}}$, Vu Tan Trao ${ }^{6}$, Marcelo Chen ${ }^{5,7,8,9}$, \\ Jason C. Huang ${ }^{4,5}$, Phung Dac Cam ${ }^{1,10}$, Yu-Ting Lin ${ }^{5 \#}$, Yi-Ming Arthur Chen ${ }^{5,11,12 \#}$ \\ ${ }^{1}$ Division of Enteric Infections, Department of Bacteriology, National Institute of Hygiene and Epidemiology, Hanoi, Vietnam; \\ ${ }^{2}$ International Health Program, National Yang-Ming University, Taipei, Taiwan; \\ ${ }^{3}$ Institute of Biomedical Sciences, Academia Sinica, Taipei, Taiwan; \\ ${ }^{4}$ Department of Biotechnology and Laboratory Science in Medicine, National Yang-Ming University, Taipei, Taiwan; \\ ${ }^{5}$ AIDS Prevention and Research Center, National Yang-Ming University, Taipei, Taiwan; \\ ${ }^{6}$ Immunology and Molecular Biology Department, National Institute of Hygiene and Epidemiology, Hanoi, Vietnam; \\ ${ }^{7}$ Department of Urology, Mackay Memorial Hospital, Taipei, Taiwan; \\ ${ }^{8}$ Mackay Medicine, Nursing and Management College, Taipei, Taiwan; \\ ${ }^{9}$ School of Medicine, Mackay Medical College, New Taipei City, Taiwan; \\ ${ }^{10}$ National Supervising Council of Professor in Medical bBranch, National Institute of Hygiene and Epidemiology, Hanoi, Vietnam; \\ ${ }^{11}$ Department of Microbiology, School of Medicine, National Yang-Ming University, Taipei, Taiwan; \\ ${ }^{12}$ Department of Microbiology, Faculty of Medicine, College of Medicine, Kaohsiung Medical University, Kaohsiung, Taiwan; \\ "Corresponding Authors: ytlin3@ym.edu.tw, arthur@ym.edu. tw
}

Received 9 September 2012; revised 7 October 2002; accepted 19 October 2012

\section{ABSTRACT}

Outbreaks of highly pathogenic avian influenza H5N1 virus have occurred in Vietnam since 2003. However, how people got avian H5N1 infection in Northern Vietnam is still unclear. We therefore performed a combination of the serologic assays H5N1 ELISA and H5 western blot to detect anti-H5N1 specific antibodies. Sera samples of 149 subjects with suspected H5N1 infection from three provinces of Northern Vietnam were collected from September 2006 to March 2007. Our results indicated that this combinational assay showed high sensitivity (100\%) and specificity (95\%) when compared with hemagglutinin inhibition (HI) assay. Fifty-one sera samples (34.2\%) contained specific antibodies against H5N1 viruses. Poultry raisers (32/77; 41.6\%) showed higher H5N1 infection rates than slaughterers $(12 / 41 ; 29.3 \%)$ and health care workers (7/31; 22.6\%). Contact history with sick or dead poultry in household or slaughter-house $(p<0.05)$ and lack of protective equipment use when in contact with dead poultry $(p<0.05)$ were risk factors found to be associated with H5N1 infection. In this study, we established an alternative sero-

${ }^{*}$ These authors contributed equally to this work.

We report no potential conflict of interest relevant to this article. logic assay for H5N1 diagnosis, and we hereby present seroepidemiologic data of H5N1 infection in Northern Vietnam.

Keywords: H5N1; ELISA; Western Blot; Hemagglutinin Inhibition; Vietnam

\section{INTRODUCTION}

The global spread of highly pathogenic avian influenza (HPAI) A H5N1 viruses in poultry and sporadic human infections are viewed as a potential pandemic threat [1]. The H5N1 virus mostly disseminates among poultry and migrating birds. Reports have indicated that humans infected with the $\mathrm{H} 5 \mathrm{~N} 1$ virus were in close contact with infected dead birds [1-3]. According to WHO's report, there have been 576 human infections and 339 deaths from 2003 to 2011 [4].

The influenza virus belongs to the Orthomyxoviridae family, which consists of enveloped viruses with a segmented single-strand RNA (negative strand). Influenza A viruses are divided into subtypes based on the antigenicity of their hemagglutinin (HA) and neuraminidase (NA) glycoproteins [5]. To date, there are 16 and 9 subtypes of HA and NA, respectively. Influenza viruses often cause annual epidemics and are responsible for several human pandemics in recent history [6]. The change of antigenicity of influenza viruses is based on antigenic drift and 
antigenic shift, the former caused by low fidelity of viral RNA polymerase and accumulating point mutations in HA and NA genes, and the latter resulting from the reassortment of gene segments between human, avian and swine influenza viruses $[7,8]$. HA proteins play a crucial role in the life cycle of the influenza A virus. The HA protein binds with its receptor expressed on the host cells and then penetrates into cell cytoplasm by mediating the fusion of the endocytosed virus membrane with the endosomal membrane. The major cell receptor of influenza virus targets is glycosylated oligosaccharides that terminate in a sialic acid (SA) residue. Influenza A viruses target primarily airway epithelial cells via $\alpha-2,3$ and $\alpha$ 2,6 linked SA receptors [9]. The avian H5N1 influenza virus was first found to infect humans, causing disease and death, in 1997 in Hong Kong [10]. Most studies indicated that H5N1-infected human cases may have resulted from close contact with these H5N1-infected sick birds, but there were some reports indicating that humanto-human transmission of $\mathrm{H} 5 \mathrm{~N} 1$ has been found and even proved in some countries [11].

At present, influenza A H5N1 viruses have been genetically separated into different clades, with the most diverse clade 2 further divided into 5 subclades. Vietnam is one of the countries with the highest number of avian influenza patients in the world. Reports indicated that influenza H5N1 outbreaks in poultry and humans in Vietnam between 2003 and 2005 were caused by clade 1 viruses [12]. Multiple sublineages of influenza subtype H5N1 viruses were epidemic in Vietnam during 20052007. Clade 2.3.4 viruses had replaced clade 1 viruses in Northern Vietnam by the end of 2007, and clade 1 viruses have been detected in Southern Vietnam [13]. Le et al. [14] indicated that before May 2007, HPAI H5N1

(a)

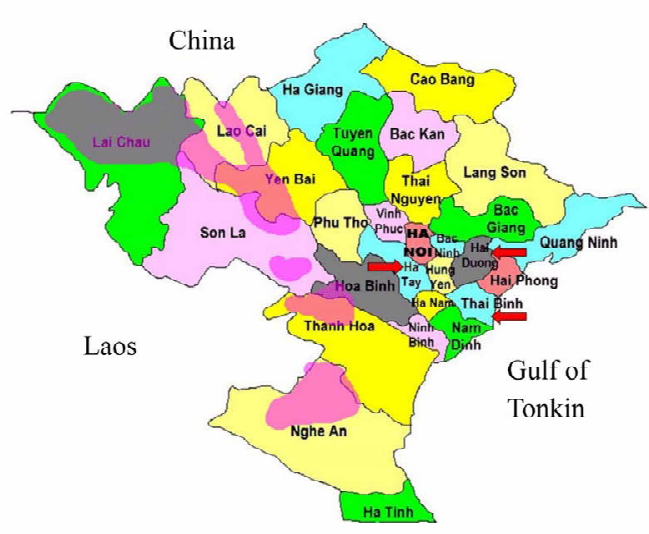

viruses isolated from poultry and humans in Northern Vietnam were consistently reported to be clade 1 viruses. The first case of human infection in Vietnam was reported in 2004. At present, only limited information about H5N1 infection to humans in Northern Vietnam is available.

Accurate and prompt diagnosis of $\mathrm{H} 5 \mathrm{~N} 1$ infection is a critical component of the disease control plan. Currently, the standard procedure for avian influenza virus detection and classification entails conventional virus isolation in embryonated eggs or MDCK cells, followed by hemagglutinin (HA) and neuraminidase (NA) subtyping using serological or RT-PCR methods [15]. For serological diagnosis, the hemagglutinin inhibition test (HI), virus neutralization test (VN), microneutralization (MN), enzyme immunoassay (EIA), and Western blot (WB) assays have been used to evaluate influenza virus-specific antibodies $[16,17]$. A recent study comparing different serological assays indicated that the combination of $\mathrm{MN}$ with WB assays or ELISA with WB assays showed maximum sensitivity and specificity in avian influenza A $\mathrm{H} 5 \mathrm{~N} 1$ virus infection [18]. To obtain a clearer picture of the H5N1 infection in humans in Northern Vietnam, we established a combination of H5N1 ELISA and H5 WB assays to screen the suspected H5N1 cases in northern Vietnam.

\section{MATERIALS AND METHODS}

\subsection{Subjects}

The serological survey was carried out in subjects living in northern Vietnam provinces, including Ha Tay, Hai Duong and Thai Binh (Figure 1(a)) from September 2006 to March 2007. In these three provinces, avian in-

(b)

\begin{tabular}{lc}
\hline Characteristics & Participants $(\mathrm{n}=149)$ \\
\hline Province & Numbers $(\%)$ \\
\hline Ha Tay & $47(31.5)$ \\
Hai Duong & $52(34.9)$ \\
Thai Binlı & $50(33.6)$ \\
& \\
Age & \\
Median, years (range) & 40.64 years \\
& $(9-76$ years $)$ \\
Occupation & \\
Health care worker & $31(20.8)$ \\
Poultry raiser & $77(51.7)$ \\
Slaughterer & $41(27.5)$ \\
\hline
\end{tabular}

Figure 1. The H5N1 outbreak provinces in Vietnam and demographic characteristics of the study participants. (a) A total of 149 serum samples were collected from Ha Tay, Hai Duong, and Thai Binh provinces (arrow) where H5N1 outbreaks occurred in 2004 and 2005; (b) The demographic data of the 149 subjects. 
fluenza A H5N1 spread to poultry in 2003-2004 and humans died of avian influenza H5N1 infection. A total of 149 participants reported to have contact history with dead avian H5N1-infected birds or with H5N1-infected patients were recruited. Avian H5N1 infection was confirmed in part of the participants by hemagglutinin inhibition (HI) or micronutralization (MN) assays, which were performed by the Immunology and Molecular Biology Department, National Institute of Hygiene and Epidemiology, Vietnam. The 20 negative control Vietnamese subjects did not have contact history with poultry or $\mathrm{H} 5 \mathrm{~N} 1$ patients and their sera did not contain antiH5N1 antibodies measured by HI and MN. In addition, 40 Taiwanese participants who were H5N1 sera negative and confirmed by $\mathrm{HI}$ and neutralization assay were also recruited in this study. Twenty Taiwanese seasonal vacinees were recruited for H5N1 and H5 WB assay specificity testing control. Blood samples were transported to Professor Vu Tan Trao's laboratory at the National Institute of Hygiene and Epidemiology (NIHE) in Vietnam and frozen at $-80^{\circ} \mathrm{C}$.

\subsection{Ethics Statement}

Ethical approval for this study was obtained from the National Institute of Hygiene and Epidemiology (NIHE) in Ha Noi. The ethics approval number is 437/QDVSDT. All the samples and survey information were obtained with informed consent from all study participants.

\subsection{H5N1 Virus Coated ELISA}

H5N1 reverse genetics (RG) strain (A/Vietnam/1203/04) coated on the 96 well plates was used to validate the humoral response. The details were described previously [19]. Briefly, individual wells of 96-well plates (PerkinElmer $^{\mathrm{TM}}$, SpectraPlate ${ }^{\mathrm{TM}}-96 \mathrm{HB}$ ) were coated with H5N1RG strain (HA titer 1:256), diluted in 1:400 with PBS, and incubated at $4{ }^{\circ} \mathrm{C}$ overnight. The next day, plates were washed three times using PBS, and then blocked with PBS containing $10 \%$ milk at $37^{\circ} \mathrm{C}$ for 1 hour. The sera collected from human and rabbit polyclonal antibodies were diluted 100 -fold with $0.05 \%$ PBST (PBS buffer contains $0.05 \%$ Tween 20 (plusone ${ }^{\circledR}$, No. 171316-01)) containing $5 \%$ milk, then incubated at $37^{\circ} \mathrm{C}$ for $1 \mathrm{~h}$. After washing five times with PBST, each well was added $100 \mu \mathrm{l}$ (1:4000 dilution) anti-human IgG Ab and (1:6000 dilution) anti-rabbit IgG Ab conjugated with horseradish peroxidase (HRP) (Amersham Biosciences) as secondary antibodies and incubated at $37^{\circ} \mathrm{C}$ for $1 \mathrm{~h}$. After five extensive washings with PBST, $200 \mu \mathrm{l} \mathrm{sub-}$ strate $(0.015 \%$ o-phenylenediamine dihydrochloride) (Sigma-Aldrich) was incubated for $30 \mathrm{~min}$ at $37^{\circ} \mathrm{C}$. Reactions were stopped by the addition of $3 \mathrm{~N} \mathrm{HCL}$; absorbance was measured with a spectrophotometer (Labsystems Multiskan Ascent Autoreader; model 354, Finland) at $492 \mathrm{~nm}$.

\subsection{H5 Hemagglutinin Glycosylated Protein Preparation}

The glycosylated HA proteins of avian influenza A H5N1 A/Thailand/1(KAN-1)/2004 strains were kindly provided by Dr. Suh-Chin Wu from the Institute of Biotechnology, National Tsing-Hua University, Hsinchu, Taiwan. The H5 recombinant proteins were prepared with a baculovirus cell-based system. Briefly, HA proteins of A/Thailand/1(KAN-1)/2004 strain were prepared by the cotransfection of baculovirus transfer vector with BaculoGold-linearized baculovirus DNA (BD Biosciences, Bedford, MA) into Spodoptera frugiperda (Sf9) cells (Invitrogen, Carlsbad, CA) using the BaculoGold transfection buffer set (BD Biosciences, Bedford, MA) and subsequently amplified in the same cells. HA was recovered from the cell supernatant by metal affinity chromatography using Ni Sepharose high-performance resin (GE Healthcare, Piscataway, NJ). Fractions containing HA were combined and subjected to ion-exchange chromatography using a MonoQ HR10/10 column (GE Healthcare, Piscataway, NJ). HA oligomers, trimers, and monomers were separated by gel filtration chromatography using a Hi-Load 16/60 Superdex 200-pg column (GE Healthcare, Piscataway, NJ).

\subsection{H5 Western Blot Assay}

The H5 glycoproteins generated from the bacovirus cell-based system mentioned above were used in the H5 WB assay. The procedures of WB were described previously [17]. H5 proteins were separated by SDS PAGE and transferred to nitrocellulose membranes (PolyScreen, PerkinElmer). Transferred antigens were incubated with the sera from participants or controls at $37^{\circ} \mathrm{C}$ for $1 \mathrm{~h}$. After three washings with PBST, NC membranes were incubated with HRP-conjugated antibodies (goat antimouse IgG, goat anti-human IgG, or goat anti-rabbit IgG) (Amersham Biosciences) for $1 \mathrm{hr}$ at $37^{\circ} \mathrm{C}$. Hybridized protein bands were visualized using the $0.05 \%$ 3-3'Diaminobenzidine detection system (SIGMA).

\subsection{Hemagglutinin Inhibition (HI) Assay}

The HI assay was performed as previously described [20]. Briefly, the serum was pretreated with receptor destroying enzyme (1:10 dilution) (Cholera filtrate, Sigma, Germany) to inactivate non-specific inhibitors. The 2fold serial diluted sera were incubated with 4 HAU H5N1-RG viruses at room temperature for $30 \mathrm{~min}$ in V-bottom 96 -well plates. $0.5 \%$ freshly prepared turkey red blood cells (RBCs) were added and then incubated at 
room temperature for $1 \mathrm{hr}$. HI titer was determined by calculation of the reciprocal of the last serum dilution which contained non-agglutinated RBCs.

\subsection{Miconeutralization (MN) Assay}

A sensitive $\mathrm{MN}$ assay was performed to detect the neutralizing capability of anti-sera; a detailed description of the procedure was previously given [17,21]. Briefly, all sera were heat-inactivated for $30 \mathrm{~min}$ at $56^{\circ} \mathrm{C}$ and serially diluted two-fold in a 96-well tissue culture plate (Becton Dickinson Labware, USA). Diluted sera $(50 \mu \mathrm{l})$ were mixed with an equal volume of influenza H5N1RG virus (100 TCID $50 / \mathrm{ml})$ and incubated for $2 \mathrm{~h}$ at $37^{\circ} \mathrm{C}$, after which $100 \mu \mathrm{l}$ of MDCK cells $\left(1.5 \times 10^{5} / \mathrm{ml}\right)$ were added to each well and incubated for $18 \mathrm{~h}$ at $37^{\circ} \mathrm{C}$. Monolayers were washed with PBS and fixed in cold $80 \%$ acetone for $10 \mathrm{~min}$. The presence of viral proteins was detected using a monoclonal antibody against the influenza A NP (1:1000 dilution) (Abcam) followed by anti-mouse-IgG conjugated HRP (1:4000 dilution) (Amersham Biosciences). Following the addition of OPD substrate, absorbance was measured at $492 \mathrm{~nm}$. All tested sera were assayed in triplicate. Neutralizing endpoints were determined using 50\% specific signal calculations as previously described [18].

\subsection{Statistical Analysis}

Information on age, gender, address, history of exposure was obtained through interviews conducted during the collection of serum samples. Data from the questionnaires were entered into a computer using Microsoft Excel 2007 and then converted to Statistical Package of Social Sciences version 17 (SPSS 17.0) for analysis. Fisher exact test was employed to analyze the data. A p value of $<0.05$ was taken as the level of statistical significance.

\section{RESULTS}

A total of 149 participants from three provinces of northern Vietnam were recruited in this study (Figure 1(a)). Their main occupations were divided into three groups: poultry raisers $(51.7 \%)$, slaughterhouse workers $(27.5 \%)$ and health care workers $(20.8 \%)$. The mean age was $40.6 \pm 13.7$ years (range, 9 - 76 years) (Figure 1(b)).

H5N1 virus coated ELISA assay (H5N1 ELISA) was established by using H5N1 reverse genetics (RG) strain (A/Vietnam/1203/04) to coat on the plate [19]. We first used H5N1-uninfected sera $(\mathrm{n}=42)$ confirmed by HI assay to determine the cut-off value of H5N1 ELISA (cut-off value $=$ mean +3 S.D). Results indicated that the cut-off O.D value was 0.958 , and results above the cutoff value were considered H5N1 seropositive (Figure 2(a)). Glycosylated HA proteins of avian influenza A
H5N1 A/Thailand/1(KAN-1)/2004 strains were prepared with baculovirus cell-based system and used for H5 western blot assay to detect anti-H5 specific antibodies (Figure 2(b)). The intact HA trimer and denatured HA proteins HA0/HA1/HA2 could be detected in the cell lystaes and culture medium in native and denaturing gels electrophoresis analyses (Figure 2(b)). Results from WB analysis of sera from $\mathrm{H} 5 \mathrm{~N} 1$ vaccinees and $\mathrm{H} 5 \mathrm{~N} 1$ seropositive subjects showed a reactive band in H5 HA (Figure 2(c), lanes 4 and 5-7). Notably, the sera from H5N1 seronegative subjects and seasonal flu vaccinee did not react with H5 HA protein in the WB (Figure 2(c), lane $8-10$ ). Further, we used $20 \mathrm{H} 5 \mathrm{~N} 1$ positive sera (form $\mathrm{H} 5 \mathrm{~N} 1$ vaccinees) and $20 \mathrm{H} 5 \mathrm{~N} 1$ negative sera to evaluate the sensitivity and specificity of our combinational assay. Figure 2(d) showed that 25 were H5N1 ELISA positive and 15 were negative. H5 WB confirmatory analysis showed that 21 were positive and 19 were negative. While H5N1 ELISA may show cross-reactivity of the antibodies against other subtypes of the influenza A virus, this cross-reactivity could be ruled out by H5 WB confirmation assay. We compared the sensitivity and specificity of H5N1 ELISA combined with H5 WB with those of the HI assay, which is considered the gold standard in clinical laboratory, and our results indicated that our combinational assay had higher sensitivity (100\%) and specificity $(95 \%)$ in detecting specific anti-H5N1 antibodies (Table 1).

The combinational assay was used to survey H5N1 infection of 149 sera samples from H5N1-suspected subjects in northern Vietnam. Results of our combinational assay indicated that 51 out of $149(34.2 \%)$ were H5N1 seropositive (Table 2). All the H5N1-suspected sera samples collected in this study were from slaughterers, poultry raisers and health care workers. We further analyzed the H5N1 infection prevalence rate in these three groups. Results indicated that poultry raisers had higher infection rate $(41.6 \%)$ than slaughterers $(29.3 \%)$ and health care workers $(22.6 \%)$. Some of the samples were also confirmed by MN or HI assays (Table 2).

We further analyzed the risk factors for H5N1 infection among these three groups (Table 3). A total of 31 serum samples were obtained from health care workers. Eleven subjects in this group were reported to have direct contact with H5N1-infected patients. Seven subjects were found to be H5N1 seropositive and 6 of them had history of exposure to H5N1-infected patients. The main risk factor in health care workers was exposure to H5N1infected patients $(\mathrm{p}<0.05, \mathrm{OR}=22.8,95 \% \mathrm{CI}=2.2$ 235) (Data not shown). Seventy-seven poultry raisers were recruited in this study. The results indicated that the presence of sick or dead poultry in households and direct contact with infected poultry were not correlated with $\mathrm{H} 5 \mathrm{~N} 1$ seropositive rates $(\mathrm{p}>0.05,95 \% \mathrm{CI}=0.9-2.4$ 
(a)

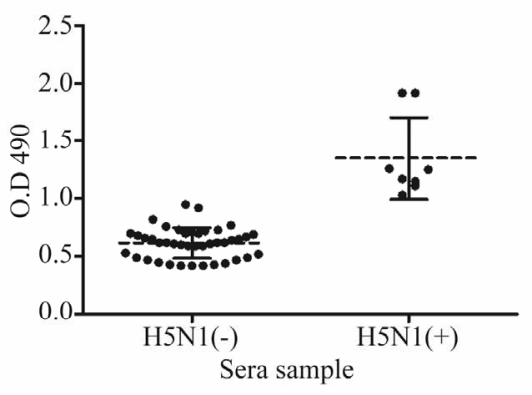

(b)

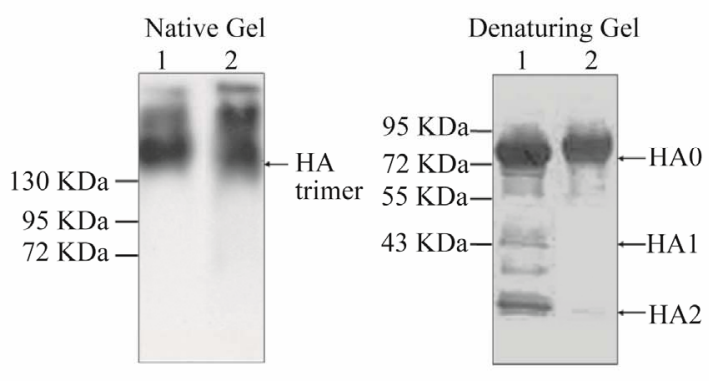

(d)

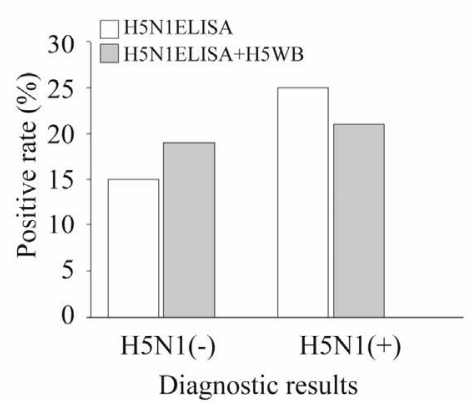

Figure 2. Establishing the H5N1 ELISA and H5 western blot. Sera samples of H5N1 uninfected persons, seasonal flu vaccinees and $\mathrm{H} 5 \mathrm{~N} 1$ vaccinees were collected for establishment and evaluation in H5N1 ELISA and H5 western blotting. (a) 42 H5N1-uninfected sera were used in H5N1 ELISA to react with the viral antigen of whole influenza H5N1 A/Vietnam/1203/04 viruses. The cut-off value was defined as the mean O.D. value plus 3 times the standard deviation (S.D.). Sera from 8 H5N1 vaccinees served as positive control; (b) H5 hemagglutinin proteins produced by baculovirus cell-based systems. The lysates (lane 1) and culture supernatant (lane 2) of Sf9 cells were collected then analyzed by native or denatured PAGE and western blotting using anti-H5N1 rabbit polyclonal antibodies to detect HA trimer and HA0/HA1/HA2 domains; (c) Representative results of H5 western blotting of sera samples reacting with H5 (A/Thailand/1(KAN-1)/2004) glycosylated hemagglutinin proteins are shown. Lanes 1 and 2: paired sera of rabbit anti-H5N1 polyclonal antibodies; lanes 3-4: paired sera of $\mathrm{H} 5 \mathrm{~N} 1$ vaccinee; lanes 5-7: sera from our subjects whose H5N1 seropositivity was confirmed by $\mathrm{HI}$ and $\mathrm{MN}$ assays; lanes 8 and 9: sera from our subjects whose H5N1 seronegativity was confirmed by $\mathrm{HI}$ and $\mathrm{MN}$ assays; lane 10: sera from seasonal flu vaccinee (seasonal flu vaccine contains H1N1 + H3N2 + Flu-B). Arrows indicate H5-HA proteins; (d) 20 sera of H5N1 vaccinees and 20 sera of $\mathrm{H} 5 \mathrm{~N} 1$ uninfected persons were used for sensitivity and specificity evaluation in our combinational serologic assays. These sera were confirmed by HI and MN assays a priori.

Table 1. Sensitivity and specificity of H5N1 ELISA combined with $\mathrm{H} 5$ western blot assay for detection of antibodies against $\mathrm{H} 5 \mathrm{~N} 1$ virus.

\begin{tabular}{ccc}
\hline & \multicolumn{2}{c}{ HI assay } \\
\cline { 2 - 3 } ELISA + WB & Positive & Negative \\
\hline Positive & $20(95.2 \%)$ & 1 \\
Negative & 0 & 19 \\
& Sensitivity: $100 \%$ & Specificity: $95 \%$ \\
\hline
\end{tabular}

Footnote: The correlation of HI and ELISA + WB was analyzed by McNemar's test $(\mathrm{p}>0.05)$.

and $0.7-8.9$ ). However, subjects who did not use protective equipment when in contact with sick or dead poultry had a significantly higher risk for H5N1 infection ( $\mathrm{p}<$ $0.05, \mathrm{OR}=2.6,95 \% \mathrm{CI}=1.01-7.2$ ). In addition, 4 poultry raisers were identified as H5N1-infected and they were reported to have direct or close contact with H5N1infected patients. Results in slaughterhouse workers indicated that the presence of sick or dying poultry in the household $(\mathrm{p}<0.05, \mathrm{OR}=2.9,95 \%$ CI $1.1-7.7)$ and direct contact with sick or dead poultry $(\mathrm{p}<0.05, \mathrm{OR}=$ $7.1,95 \%$ CI 1.3 - 38.3) were risk factors for H5N1 infection. Moreover, the lack of protective equipment (such as masks, gloves, boots) during the slaughter process also increased the risk of $\mathrm{H} 5 \mathrm{~N} 1$ infection $(\mathrm{p}<0.05, \mathrm{OR}=5.2$, $95 \% \mathrm{CI}=1.3-22.4$ ).

\section{DISCUSSION}

Since the H5N1 outbreak in Hong Kong in 1997 [22], multiple serological methods have been developed in an effort to maximize the sensitivity and specificity for detecting H5N1-specific antibody responses. Presently, 
Table 2. Serologic combinational diagnosis of H5N1-suspected cases in Northern Vietnam.

\begin{tabular}{|c|c|c|c|c|c|}
\hline \multirow{2}{*}{ Occupation } & \multirow{2}{*}{ Sample number } & \multicolumn{4}{|c|}{ Positive (\%) } \\
\hline & & \multicolumn{2}{|c|}{ ELISA + WB } & \multirow{2}{*}{$\frac{\mathrm{MN}}{\mathrm{N} / \mathrm{A}}$} & \multirow{2}{*}{$\begin{array}{c}\mathrm{HI} \\
7(22.6 \%)\end{array}$} \\
\hline Health care worker & 31 & 7 & $(22.6 \%)$ & & \\
\hline Poultry raiser & 77 & 32 & $(41.6 \%)$ & $32(41.6 \%)$ & N/A \\
\hline Slaughterer & 41 & 12 & $(29.3 \%)$ & $12(29.3 \%)$ & $11(26.8 \%)$ \\
\hline Total & 149 & 51 & $(34.2 \%)$ & N/A & N/A \\
\hline
\end{tabular}

Footnote: ELISA indicates H5N1 virus coated ELISA assay; WB indicates H5 western blot assay; MN indicates microneutralization assay; HI indicates hemagglutinin inhibition assay; N/A indicates non-available.

Table 3. Risk factors analyses among different occupational groups of our collected sample.

\begin{tabular}{|c|c|c|c|c|c|c|}
\hline \multirow[b]{2}{*}{ Risk factors } & \multicolumn{3}{|c|}{$\begin{array}{l}\text { H5N1 Serodiagnosis among Poultry raiser } \\
\qquad(\mathrm{n}=77)\end{array}$} & \multicolumn{3}{|c|}{$\begin{array}{c}\text { H5N1 Serodiagnosis among Slaughterhouse } \\
\text { workers }(n=41)\end{array}$} \\
\hline & $+(n=32)$ & $-(n=45)$ & OR $(95 \% \mathrm{CI})$ & $+(\mathrm{n}=12)$ & $-(n=29)$ & OR $(95 \% \mathrm{CI})$ \\
\hline \multicolumn{7}{|c|}{ Household had dead poultry due to the disease } \\
\hline No & $14(33.3 \%)$ & $28(66.7 \%)$ & 1.0 (Ref) & $6(20.0 \%)$ & $24(80.0 \%)$ & 1.0 (Ref) \\
\hline Yes & $18(51.4 \%)$ & $17(48.6 \%)$ & $1.5(0.9-2.4)$ & $6(54.5 \%)$ & $5(45.5 \%)$ & $2.9(1.1-7.7)^{*}$ \\
\hline \multicolumn{7}{|c|}{ Direct contact with poultry died from the disease } \\
\hline No & $14(38.9 \%)$ & $22(61.1 \%)$ & 1.0 (Ref) & $3(15.8 \%)$ & $16(84.2 \%)$ & $1.0($ Ref) \\
\hline Yes & $18(43.9 \%)$ & $23(56.1 \%)$ & $0.8(0.7-8.9)$ & $10(45.5 \%)$ & $12(54.5 \%)$ & $7.1(1.3-38.3)^{*}$ \\
\hline \multicolumn{7}{|c|}{ Protective equipment when in contact with dead poultry } \\
\hline Yes & $25(49.0 \%)$ & $26(51.0 \%)$ & 1.0 (Ref) & $8(50.0 \%)$ & $8(50 \%)$ & 1.0 (Ref) \\
\hline No & $7(26.9 \%)$ & $19(73.1 \%)$ & $2.6(1.1-7.2)^{*}$ & $4(16.0 \%)$ & $21(84.0 \%)$ & $5.2(1.3-22.4)^{*}$ \\
\hline \multicolumn{7}{|c|}{ Direct contact with H5N1-infected patients } \\
\hline No & $28(40.0 \%)$ & $42(60.0 \%)$ & 1.0 (Ref) & N/A & & \\
\hline Yes & $4(57.1 \%)$ & $3(42.9 \%)$ & $2.0(0.4-9.6)$ & & & \\
\hline
\end{tabular}

N/A: Not available; ${ }^{*} \mathrm{p}<0.05$.

serological assays used clinically to detect influenza A virus include HI, ELISA, WB and MN assays. The HI and viral neutralization assay are considered the "gold standard" for serologic diagnosis of infection with human influenza viruses $[23,24]$. ELISA is popular and widely used in clinical virological laboratories due to its ease and speed, but it sometimes shows high false positivity owing to cross-reactivity to other types of influenza viruses. WB is often used as a second line or confirmatory assay because it is too labor-intensive to screen large samples. In this study, we used H5N1 ELISA for initial screening and H5 WB for confirmation. This combination showed high sensitivity (100\%) and specificity (95\%). Recently, Rowe et al. [18] indicated that when combined with a confirmatory H5-specific WB test, the specificities of both ELISA and MN assays improved. Their results indicated that maximum sensitivity $(80 \%)$ and specificity $(96 \%)$ for the detection of anti-H5 antibody in adults aged 18 to 59 years were achieved by using the $\mathrm{MN}$ assay combined with WB. Maximum sensitivity $(100 \%)$ and specificity $(100 \%)$ in detecting anti-H5 antibody in sera obtained from children less than 15 years of age were achieved by using ELISA combined with WB. These findings are in agreement with those of our study.

Previously, researchers suggested the use of a highly purified baculovirus-expressed H5 HA protein from $\mathrm{A} /$ Hongkong/156/97 virus (clade 3 ) in a confirmatory WB assay [24-26]. In this study, a highly purified baculovirus-expressed H5 HA protein from A/Thailand/ KAN-1/2004 strain (clade 1) was produced for the confirmatory WB assay. Recent studies indicated that before May 2007, highly pathogenic avian H5N1 viruses isolated from poultry and humans in northern Vietnam were reported to be clade 1 viruses [14]. Accordingly, we believe that most of the subjects infected with H5N1 were mainly infected with clade 1 virus. We further compared the amino acid identity between clade 1 and 2.3.4 and the results showed $95.4 \%$ - 96.6\% sequence identity between these two clades. It was therefore reasonable to select a H5N1 clade 1 virus (A/Vietnam/1204/03 and A/Thailand/KAN-1/2004) as reactive antigen to set up the H5N1 ELISA and H5 WB.

The seroprevalence of H5N1 infection in different oc- 
cupational groups has been continuously surveyed to better understand the epidemiology of human $\mathrm{H} 5 \mathrm{~N} 1$ infection [27-29]. In this study, H5N1-suspected subjects were divided according to their occupation into poultry raisers, slaughterhouse workers and health care workers. Our results showed a higher seropositive rate in poultry raisers and slaughterers (41.6\% and 29.3\%). In a cohort of 293 Hong Kong government workers participating in a poultry culling operation and 1525 poultry workers, Bridge et al. [25] found that approximately $10 \%$ of poultry workers had anti-H5 antibodies. They suggested that increased exposure to poultry, such as by butchering or exposure to ill poultry, was associated with the presence of anti-H5 antibody [25]. Suatana et al. indicated that poultry raisers handling and slaughtering sick birds were at a higher risk of avian H5N1 infection. They observed disease transmission from poultry to poultry, but not from poultry to humans [30]. The facts that the majority of our subjects lived in traditional Vietnamese communities raising poultry in nearby areas and that continuous H5N1 outbreaks occurred in northern Vietnam may have contributed to increased risks of H5N1 infection. Our results also indicated that 7 of $31(22.5 \%)$ health care workers were $\mathrm{H} 5 \mathrm{~N} 1$ seropositive. These health care workers were found to be in direct or indirect contact with H5N1 patients (data not shown). Most cases of H5N1 influenza virus infection are believed to occur through bird-to-human transmission. A few isolated cases of suspected human-to-human transmission have been reported [15,31]. A study in Hong Kong provided evidence that health care workers were infected with H5N1 via exposure to H5N1-infected patients [32]. We therefore suggest that $\mathrm{H} 5 \mathrm{~N} 1$ human-to-human transmission is possible and may increase the chances for the emergence of a novel influenza virus with pandemic threats.

Risk factor analysis showed that a significantly higher risk of $\mathrm{H} 5 \mathrm{~N} 1$ infection was observed in slaughterhouse workers in direct contact with dead poultry $(\mathrm{OR}=2.9$, $95 \% \mathrm{CI}=1.1-7.7)$ and this was consistent with Dinh et al.'s study [33] in Vietnam (OR $=7.41,95 \% \mathrm{CI}=2.7$ 59) and Areechokchai et al.'s study [34] in Thailand (OR $=29,95 \% \mathrm{CI}=2.7-308.2$ ). In our study, poultry raisers in direct contact with sick or dead poultry were not at an increased risk. However, our results indicated that healthy poultries may also be an important source of viral transmission as $14 \mathrm{H} 5 \mathrm{~N} 1$ seropositive cases did not have any previous contact with sick or dead poultry (Table 3). Our results indicated that using protective equipment is important for $\mathrm{H} 5 \mathrm{~N} 1$ prevention. These findings are similar to those reported by Mounts et al. [35] from a study of markets selling raw poultries in Hong Kong. H5N1 seropositive rates were correlated with the use of protective equipment when in contact with sick or dead poultry. This correlation was statistically significant in both poul- try raisers and slaughterhouse workers $(\mathrm{p}<0.05)$. We suggest that the use of protective equipment when touching sick or dead poultry is extremely important.

Our study revealed a high prevalence of $\mathrm{H} 5 \mathrm{~N} 1$ seropositivity in northern Vietnam. This may be due to a lack of awareness of the dangers of the disease by Vietnamese people who were in contact with sick or dead poultry, as most rural households in Vietnam breed small poultry or have poultry cages near their houses. This may increase the risk of the virus spreading in the community. Similar findings and situations were also reported in Cambodia [36], Nigeria [37] and Thailand [38]. Combined, we suggest that preventive measures and education are therefore necessary to decrease the risk of viral spread in the community. These data are valuable for influenza H5N1 virus research, diagnosis and prevention.

\section{ACKNOWLEDGEMENTS}

The authors wish to thank Prof. Vu Tan Trao of the National Institute of Hygiene and Epidemiology, Hanoi, Vietnam for providing the sera sample and for discussions regarding protocols, and Prof. Suh-Chin $\mathrm{Wu}$ of the Institute of Biotechnology, National Tsing-Hua University, Hsinchu, Taiwan for providing the Glycosylated H5-HA proteins. This work was supported by grants from the Republic of China National Science Council (NSC-98-2321-B-010-004).

\section{REFERENCES}

[1] Pawitan, J.A. (2007) Human H5N1 influenza. The New England Journal of Medicine, 356, 1376-1377. doi:10.1056/NEJMc063637

[2] Dinh, P.N., Tien, N.T., Hien, N.T., Mai Le, T.Q., Phong Le, H., Tuan Le, V., Van Tan, H., Nguyen, N.B., Van Tu, P. and Phuong, N.T. (2006) Risk factors for human infection with avian influenza A H5N1, Vietnam, 2004. Emerging Infectious Diseases Journal, 12, 1841-1847. doi:10.3201/eid1212.060829

[3] Subbarao, K., Klimov, A., Katz, J., Regnery, H., Lim, W., Hall, H., Perdue, M., Swayne, D., Bender, C., Huang, J., et al. (1998) Characterization of an avian influenza A (H5N1) virus isolated from a child with a fatal respiratory illness. Science, 279, 393-396. doi:10.1126/science. 279.5349 .393

[4] Wang, T.T., Parides, M.K. and Palese, P. (2012) Seroevidence for H5N1 influenza infections in humans: Metaanalysis. Science, 335, 1463. doi:10.1126/science. 1218888

[5] Renegar, K.B. (1992) Influenza virus infections and immunity: A review of human and animal models. Laboratory Animal Science, 42, 222-232.

[6] Silverstein, G. (2006) Preparing for pandemic influenza. Lancet, 367, 1239-1240. doi:10.1016/S0140-6736(06)68537-1

[7] Pontoriero, A.V., Baumeister, E.G., Campos, A.M., Savy, V.L., Lin, Y.P. and Hay, A. (2003) Antigenic and genomic relation between human influenza viruses that circulated 
in Argentina in the period 1995-1999 and the corresponding vaccine components. Journal of Clinical Virology, 28, 130-140. doi:10.1016/S1386-6532(02)00274-3

[8] Carrat, F. and Flahault, A. (2007) Influenza vaccine: The challenge of antigenic drift. Vaccine, 25, 6852-6862. doi:10.1016/j.vaccine.2007.07.027

[9] Gagneux, P., Cheriyan, M., Hurtado-Ziola, N., van der Linden, E.C., Anderson, D., McClure, H., Varki, A. and Varki, N.M. (2003) Human-specific regulation of alpha 2-6-linked sialic acids. The Journal of Biological Chemistry, 278, 48245-48250. doi:10.1074/jbc.M309813200

[10] Lavanchy, D. (1998) The WHO update on influenza A (H5N1) in Hong Kong. Euro Surveillance, 3, 23-25.

[11] Ligon, B.L. (2005) Avian influenza virus H5N1: A review of its history and information regarding its potential to cause the next pandemic. Seminars in Pediatric Infectious Diseases, 16, 326-335. doi:10.1053/j.spid.2005.07.002

[12] Abdel-Ghafar, A.N., Chotpitayasunondh, T., Gao, Z., Hayden, F.G., Nguyen, D.H., de Jong, M.D., Naghdaliyev, A., Peiris, J.S., Shindo, N., Soeroso, S., et al. (2008) Update on avian influenza A (H5N1) virus infection in humans. The New England Journal of Medicine, 358, 261273. doi:10.1056/NEJMra0707279

[13] Dung Nguyen, T., Vinh Nguyen, T., Vijaykrishna, D., Webster, R.G., Guan, Y., Malik Peiris, J.S. and Smith, G.J., (2008) Multiple sublineages of influenza A virus (H5N1), Vietnam, 2005-2007. Emerging Infectious Diseases Journal, 14, 632-636. doi:10.3201/eid1404.071343

[14] Le, M.T., Wertheim, H.F., Nguyen, H.D., Taylor, W., Hoang, P.V., Vuong, C.D., Nguyen, H.L., Nguyen, H.H., Nguyen, T.Q., Nguyen, T.V., et al. (2008) Influenza A H5N1 clade 2.3.4 virus with a different antiviral susceptibility profile replaced clade 1 virus in humans in northern Vietnam. PLoS One, 3, e3339. doi:10.1371/journal.pone.0003339

[15] Beigel, J.H., Farrar, J., Han, A.M., Hayden, F.G., Hyer, R., de Jong, M.D., Lochindarat, S., Nguyen, T.K., Nguyen, T.H., Tran, T.H., et al. (2005) Avian influenza A (H5N1) infection in humans. The New England Journal of Medicine, 353, 1374-1385. doi:10.1056/NEJMra052211

[16] Imai, M., Ninomiya, A., Minekawa, H., Notomi, T., Ishizaki, T., Van Tu, P., Tien, N.T., Tashiro, M. and Odagiri, T. (2007) Rapid diagnosis of H5N1 avian influenza virus infection by newly developed influenza H5 hemagglutinin gene-specific loop-mediated isothermal amplification method. Journal of Virological Methods, 141, 173-180. doi:10.1016/j.jviromet.2006.12.004

[17] Wang, S., et al. (2009) Generating and characterizing monoclonal and polyclonal antibodies against avian H5N1 hemagglutinin protein. Biochemical and Biophysical Research Communications, 382, 691-696. doi:10.1016/j.bbrc.2009.03.119

[18] Rowe, T., et al. (1999) Detection of antibody to avian influenza A (H5N1) virus in human serum by using a combination of serologic assays. Journal of Clinical Microbiology, 37, 937-943.

[19] Wang, S., et al. (2009) Generating and characterizing monoclonal and polyclonal antibodies against avian $\mathrm{H} 5 \mathrm{~N} 1$ hemagglutinin protein. Biochemical and Biophysical Research Communications, 382, 691-696. doi:10.1016/j.bbrc.2009.03.119

[20] Grund, S., et al. (2010) Comparison of hemagglutination inhibition assay, an ELISA-based micro-neutralization assay and colorimetric microneutralization assay to detect antibody responses to vaccination against influenza A H1N1 2009 virus. Journal of Virological Methods, 171, 369-373. doi:10.1016/j.jviromet.2010.11.024

[21] Kayali, G., et al. (2008) Testing human sera for antibodies against avian influenza viruses: Horse RBC hemagglutination inhibition vs. microneutralization assays. Journal of Clinical Virology, 43, 73-78. doi:10.1016/j.jcv.2008.04.013

[22] (1997) Isolation of avian influenza A (H5N1) viruses from humans-Hong Kong, May-December 1997. Morbidity and Mortality Weekly Report, 46, 1204-1207.

[23] Hinshaw, V.S., et al. (1981) Replication of avian influenza A viruses in mammals. Infection and Immunity, $\mathbf{3 4}$ 354-361.

[24] Rowe, T., et al. (1999) Detection of antibody to avian influenza A (H5N1) virus in human serum by using a combination of serologic assays. Journal of Clinical Microbiology, 37, 937.

[25] Bridges, C.B., et al. (2002) Risk of influenza A (H5N1) infection among poultry workers, Hong Kong, 1997-1998. The Journal of Infectious Diseases, 185, 1005-1010. doi: $10.1086 / 340044$

[26] Katz, J.M., et al. (1999) Antibody response in individuals infected with avian influenza A (H5N1) viruses and detection of anti-H5 antibody among household and social contacts. The Journal of Infectious Diseases, 180, 17631770. doi:10.1086/315137

[27] WHO (2005) Recommended laboratory test to identify avian influenza A virus in specimens from humans. World Health Organization, June-September Technical Working Group, Geneva. http://www.cdc.gov.tw/downloadfile.aspx?fid=FCE2E4E 34EB2CCE3

[28] Schultsz, C., et al. (2005) Avian influenza H5N1 and healthcare workers. Emerging Infectious Diseases Journal, 11, 1158-1159. doi:10.3201/eid1107.050070

[29] Beigel, J., et al. (2005) Avian influenza A (H5N1) infection in humans. The New England Journal of Medicine, 353, 1374. doi:10.1056/NEJMra052211

[30] Sultana, R., et al. (2012) Bangladeshi backyard poultry raisers' perceptions and practices related to zoonotic transmission of avian influenza. Journal of Infection in Developing Countries, 6, 156-165.

[31] Lukrafka, J.L., et al. (2007) Determining risk factors for infection with influenza A (H5N1). Emerging Infectious Diseases Journal, 13, 955-956. doi:10.3201/eid1306.070025

[32] Buxton Bridges, C.K.J., et al. (2000) Risk of influenza A (H5N1) infection among health care workers exposed to patients with influenza A (H5N1), Hong Kong. The Journal of Infectious Diseases, 181, 334-348. 
[33] Dinh, P.N., et al. (2006) Risk factors for human infection with avian influenza A H5N1, Vietnam, 2004. Emerging Infectious Diseases Journal, 12, 1841-1847. doi:10.3201/eid1212.060829

[34] Areechokchai, D., et al. (2006) Investigation of avian influenza (H5N1) outbreak in humans-Thailand, 2004. Morbidity and Mortality Weekly Report, 55, 3-6.

[35] Mounts, A.W., et al. (1999) Case-control study of risk factors for avian influenza A (H5N1) disease, Hong Kong, 1997. The Journal of Infectious Diseases, 180, 505-508. doi:10.1086/314903

[36] Vong, S., Coghlan, B., Mardy, S., Holl, D., Seng, H., Ly, S., Miller, M., Buchy, P., Froehlich, Y. and Dufourcq, J.
(2006) Low frequency of poultry-to-human H5N1 virus transmission, southern Cambodia, 2005. Emerging Infectious Diseases Journa, 12, 6-424.

[37] Ortiz, J., et al. (2007) Lack of evidence of avian to human transmission of avian influenza A (H5N1) virus among poultry workers, Kano, Nigeria, 2006. The Journal of Infectious Diseases, 196, 1685-1691. doi:10.1086/522158

[38] Apisarnthanarak, A., et al. (2005) Seroprevalence of antiH5 antibody among Thai health care workers after exposure to avian influenza (H5N1) in a tertiary care center. Clinical Infectious Diseases, 40, e16-e18. doi:10.1086/427034 